\title{
Noncardiogenic Pulmonary Edema in Low-dose Oral Methotrexate Therapy
}

\author{
Noriko IIKUNI*, Sumiko IWAMI, Shogo KASAI and Hitoshi TOKUDA
}

\begin{abstract}
In the past two decades, low-dose methotrexate (MTX) has been widely used in the treatment of rheumatoid arthritis (RA). As adverse effects, various types of pulmonary toxicity have been reported with this therapy. We report a case of MTX-induced noncardiogenic pulmonary edema in a 35-year-old woman. MTX used in high dose for anti-cancer therapy is known to cause noncardiogenic pulmonary edema. However, there are no previous reports of noncardiogenic pulmonary edema caused by low-dose MTX therapy. This report suggests that patients receiving oral weekly, low-dose MTX may be at risk for the development of noncardiogenic pulmonary edema.
\end{abstract}

(Internal Medicine 43: 846-851, 2004)

Key words: MTX-induced lung injury, noncardiogenic pulmonary edema, low-dose methotrexate therapy

\section{Introduction}

Since its approval by the US Food and Drug Administration for the treatment of rheumatoid arthritis (RA) in 1988 (1), low-dose methotrexate (MTX) treatment has been widely used as an effective treatment for rheumatoid arthritis. MTX can cause pulmonary toxicity, the first of which was observed in the treatment of childhood leukemia in 1969 (2), and later in other diseases, but for a while it was believed that pulmonary toxicity would only occur when the weekly dose was higher than $20 \mathrm{mg}$ (3). In 1983, pneumonitis was first reported during low-dose MTX treatment for RA $(4,5)$, and since then, there have been numerous reports of MTX-induced pulmonary toxicity in low-dose MTX therapy.
Pulmonary toxicity associated with MTX use includes acute interstitial pneumonitis, interstitial fibrosis, pleuritis and pleural effusion, pulmonary nodules, and noncardiogenic pulmonary edema. Acute interstitial pneumonitis is the most common, followed by interstitial fibrosis; whereas the latter three conditions are quite rare. All pulmonary conditions have been reported in low-dose MTX therapy for RA, except for noncardiogenic pulmonary edema, which has only been seen in patients receiving high-dose MTX for the treatment of malignancies (6-9).

We report a rare case of noncardiogenic pulmonary edema caused by low-dose MTX therapy which occurred in a young woman with rheumatoid arthritis.

\section{Case Report}

A 35-year-old woman with rheumatoid arthritis was admitted to our hospital because of dry cough, fever and rapidly progressive dyspnea.

She had been diagnosed as rheumatoid arthritis (Steinbrocker Stage III, Class 2) in 1999. Her therapeutic regimen included oral corticosteroids, non-steroidal anti-inflammatory drugs (NSAIDs), and sulfasalazine, all of which had been started at the time of diagnosis. The patient did not respond well to this treatment, despite increases in doses of all the medications. In January 2001, the patient was started on 6 $\mathrm{mg} /$ week of MTX. A year later, the dose was increased to 8 $\mathrm{mg} /$ week, and the dose was progressively increased during the course of the year to a final dose of $15 \mathrm{mg} /$ week in July 2002. Her last dose of MTX was October 7, 2002.

The patient started to complain of nonproductive cough in the beginning of September 2002, with no other symptoms at that time. On October 12, she developed a fever of $40^{\circ} \mathrm{C}$ and complained of dyspnea. She was prescribed azithromycin 0.5 g/day at a clinic, but her symptoms worsened, and she was referred and admitted to our hospital three days later.

On admission, the patient's temperature was $39.6^{\circ} \mathrm{C}$,

From the Department of Internal Medicine, Social Insurance Central General Hospital, Tokyo. *Present address: Institute of Rheumatology, Tokyo Women's Medical University, Tokyo

Received for publication October 31, 2003; Accepted for publication May 17, 2004

Reprint requests should be addressed to Dr. Noriko Iikuni, Institute of Rheumatology, Tokyo Women's Medical University, 10-22 Kawada-cho, Shinjukuku, Tokyo 162-0054 
Methotrexate-induced Lung Injury

Table 1. Laboratory Data on Admission

\begin{tabular}{|c|c|c|c|}
\hline \multicolumn{2}{|l|}{ Hematology } & \multicolumn{2}{|l|}{ Serology } \\
\hline WBC & $7,010 / \mu \mathrm{l}$ & ESR & $17 \mathrm{~mm} / \mathrm{h}$ \\
\hline Neu & $73 \%$ & CRP & $13.4 \mathrm{mg} / \mathrm{dl}$ \\
\hline Ly & $10 \%$ & $\mathrm{IgG}$ & $795 \mathrm{mg} / \mathrm{dl}$ \\
\hline Eo & $1 \%$ & $\operatorname{Ig} \mathrm{A}$ & $177 \mathrm{mg} / \mathrm{dl}$ \\
\hline $\mathrm{RBC}$ & $434 \times 10^{4} / \mu 1$ & $\operatorname{IgM}$ & $124 \mathrm{mg} / \mathrm{dl}$ \\
\hline $\mathrm{Hb}$ & $14.6 \mathrm{~g} / \mathrm{dl}$ & RAPA & $160 \times$ \\
\hline Plt & $15.4 \times 10^{4} / \mu \mathrm{l}$ & ANA & $40 \times$ \\
\hline \multirow[t]{2}{*}{ CD4+Lym } & $69 \%$ & KL-6 & $332 \mathrm{U} / \mathrm{ml}$ \\
\hline & & $\beta$-D glucan & $21.5 \mathrm{pg} / \mathrm{ml}$ \\
\hline \multicolumn{2}{|c|}{ Blood chemistry } & $\mathrm{CMV}-\mathrm{Ab}$ & $(-)$ \\
\hline $\mathrm{TP}$ & $5.8 \mathrm{~g} / \mathrm{dl}$ & & \\
\hline Alb & $3.5 \mathrm{~g} / \mathrm{dl}$ & Blood MTX level & $<0.03 \mu \mathrm{mol} / l$ \\
\hline AST & $48 \mathrm{IU} / l$ & & \\
\hline ALT & $45 \mathrm{IU} / l$ & \multicolumn{2}{|c|}{ Arterial blood gas analysis } \\
\hline $\mathrm{LDH}$ & $577 \mathrm{IU} / l$ & $\mathrm{pH}$ & 7.404 \\
\hline ALP & $165 \mathrm{IU} / l$ & $\mathrm{PaCO}_{2}$ & 39.6 Torr \\
\hline CK & $183 \mathrm{IU} / l$ & $\mathrm{PaO}_{2}$ & 62.5 Torr \\
\hline BUN & $15 \mathrm{mg} / \mathrm{dl}$ & & \\
\hline Cre & $0.6 \mathrm{mg} / \mathrm{dl}$ & & \\
\hline $\mathrm{Na}$ & $138 \mathrm{mEq} / \mathrm{l}$ & & \\
\hline $\mathrm{K}$ & $4.3 \mathrm{mEq} / \mathrm{l}$ & & \\
\hline $\mathrm{Cl}$ & $105 \mathrm{mEq} / \mathrm{l}$ & & \\
\hline
\end{tabular}

TP: total protein, Alb: albumin, AST: aspartate aminotransferase, ALT: alanine aminotransferase, LDH: lactate dehydrogenase, ALP: alkaline phosphatase, CK: creatine kinase, BUN: blood urea nitrogen, ESR: erythrocyte sedimentation rate, CRP: C-reactive protein, ANA: antinuclear antibodies, CMV-Ab: cytomegalovirus antibody.

blood pressure 110/78 $\mathrm{mmHg}$, pulse $92 / \mathrm{min}$, and respiration $23 / \mathrm{min}$. The patient appeared to be in respiratory distress. No rash or lymphadenopathy was noted on physical examination. The jugular vein was not dilated. Fine crackles were heard in both lungs. The heart sounds were normal, and the abdomen was benign. There was no peripheral edema, tenderness of calves, or digital clubbing, and the peripheral pulses were strong. Neurological examination was unremarkable.

The results of laboratory tests on admission are shown in Table 1. Complete blood count showed no signs of anemia or thrombocytopenia, and the white cell count was $7,010 / \mu 1$. Although IgG levels were slightly decreased, the CD4+ lymphocyte count was 484, showing no evidence of immunosuppression caused by MTX. LDH was elevated to $577 \mathrm{IU} / l$, but concentrations of serum KL-6 were within normal range. CRP was $13.4 \mathrm{mg} / \mathrm{dl}$ and $\beta$-D glucan was slightly elevated, indicating a possibility of infection, but stains and cultures for bacteria, fungus, acid-fast organisms and stains for Pneumocystis carinii were negative. Sputum Pneumocystis carinii PCR was also negative. At 2 liters per minute of oxygen, nasal cannula, $\mathrm{PaO}_{2}$ was 62.5 Torr and $\mathrm{PaCO}_{2}$ was 39.6 Torr, indicating severe hypoxemia. Bronchoalveolar lavage fluid obtained on hospital day two showed an increase in total cell count of $8.7 \times 10^{5} / \mathrm{ml}$ with marked lymphocytosis (81\%), and CD4 to CD8 T-cell ratio
Table 2. Laboratory Data Obtained with Bronchoalveolar Lavage Fluid (BALF)

$\begin{array}{lc}\text { BALF } & \\ \text { Recovery rate } & 67 \% \\ \text { TCC } & 8.7 \times 10^{5} / \mathrm{ml} \\ \text { Neu } & 1 \% \\ \text { Ly } & 81 \% \\ \text { Eo } & 4 \% \\ \text { M } \phi & 14 \% \\ \text { CD } 4 / 8 \text { ratio } & 8.05 \\ \text { Cytology } & \text { Class II } \\ \text { Stains, cultures } & \text { negative } \\ \text { TB-PCR } & \text { negative } \\ \text { P. carinii } \text { PCR } & \text { negative }\end{array}$

TCC: total cell count, Neu: neutrophils, Ly: lymphocytes, Eo: eosinophils, M $\phi$ : macrophages, TB-PCR: tuberculosis polymerase chain reaction, $P$. carinii PCR: Pneumocystis carinii polymerase chain reaction.

was strikingly elevated to 8.05 (Table 2).

A chest radiograph a year earlier showed no significant findings, while the chest radiograph at the time of admission revealed bilateral, diffuse, hazy opacities in the middle and upper lung fields (Fig. 1). There was no pleural effusion, and 

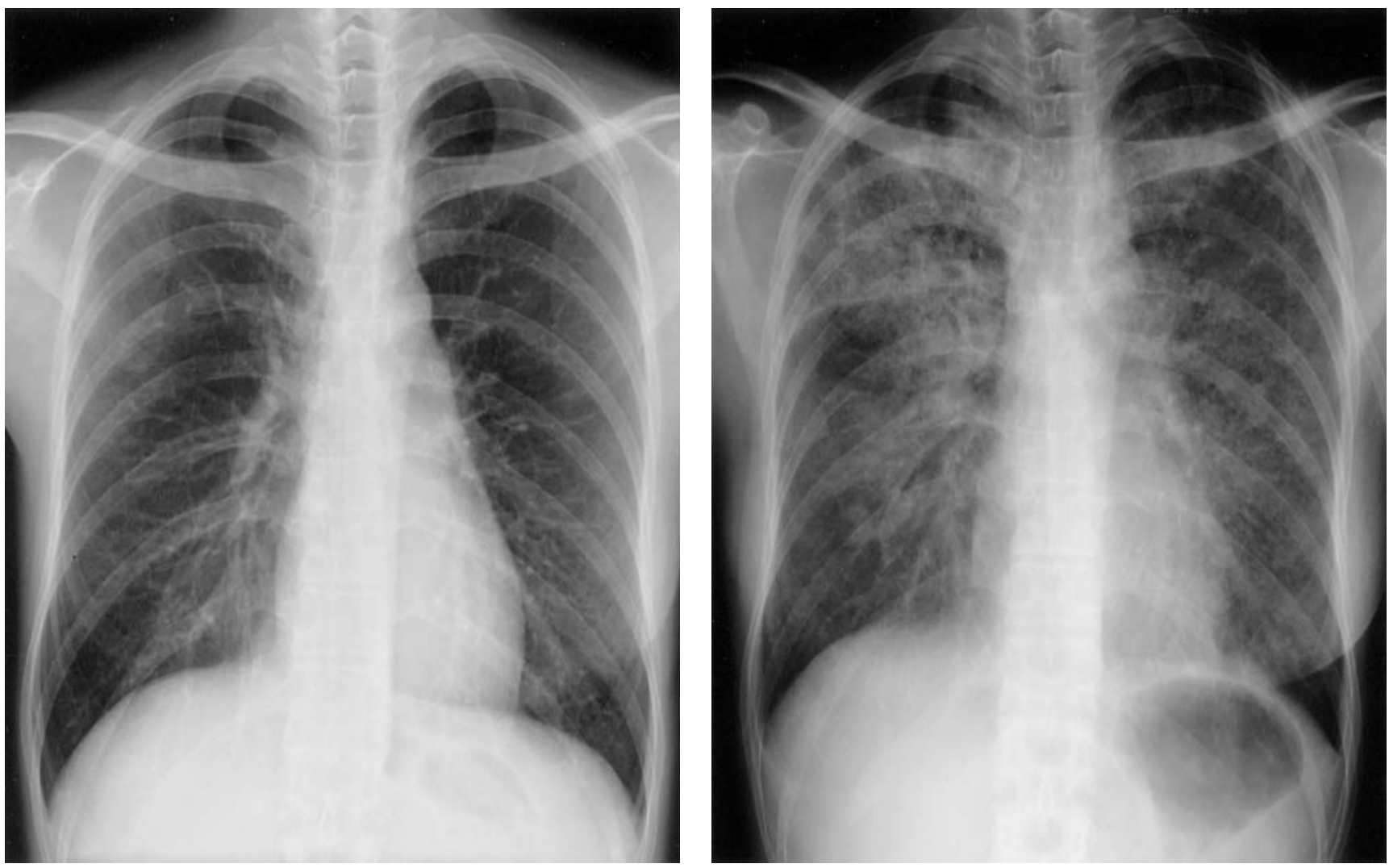

Figure 1. Chest radiograph one year before admission (left). Chest radiograph at time of admission reveals bilateral, diffuse, hazy opacities in the middle and upper lung fields (right).

the size of the heart was normal. A computed tomographic (CT) scan of the thorax revealed bilateral areas of ground glass attenuation with areas of consolidation (Fig. 2). These opacities were observed diffusely from central to peripheral zones of the lungs reaching to the thoracic wall, but seemed to be most prominent in the perihilar area. No pleural effusion, honeycombing or architectural distortions were observed.

From the patient's clinical course, laboratory data, and radiological findings, she was diagnosed as having MTXinduced lung injury. MTX was immediately discontinued. On hospital day one, the patient was administered $500 \mathrm{mg}$ of intravenous (i.v.) methylprednisolone. Despite this treatment, the patient's hypoxemia worsened the following day $\left(\mathrm{PaO}_{2}\right.$ was 62 Torr at 11 liters per minute of oxygen, face mask), and the patient was intubated and mechanical ventilation was started (Fig. 3A). She received 3 days of i.v. methylprednisolone $1,000 \mathrm{mg} / \mathrm{day}$ and diuretics. On hospital day four, the patient's respiratory condition dramatically improved, and she was taken off the ventilator. She recovered from hypoxemia, and the bilateral opacities on chest radiograph had completely resolved four days later (Fig. 3B). After two more days of $125 \mathrm{mg} /$ day i.v. methylprednisolone, the patient was switched to her pre-hospitalization cortico- steroid dose of $7.5 \mathrm{mg} / \mathrm{day}$. Her symptoms completely disappeared, and there were no signs of relapse. At the time of discharge seven days later, her $\mathrm{PaO}_{2}$ was 93.0 Torr at room air (Fig. 4).

\section{Discussion}

Methotrexate (MTX) is one of the most commonly used disease-modifying drugs for rheumatoid arthritis (RA). Its efficacy is well known, as is its side effects, especially pulmonary, hematologic, and hepatic toxicities. Although, the precise mechanism by which MTX results in pulmonary injury is unknown, pulmonary complications of MTX may cause serious implications and are life-threatening.

The five clinical pulmonary syndromes associated with MTX treatment are acute interstitial pneumonitis (hypersensitivity lung reaction), interstitial fibrosis, pleuritis or pleural effusions, pulmonary nodules, and noncardiogenic pulmonary edema. Pneumonitis due to MTX usually presents either acutely or subacutely with cough, fever, and dyspnea, and since no clinical features are specific or diagnostic for any MTX-induced pulmonary disease, it is difficult to differentiate between these various types of pulmonary manifestations initially. Observations of clinical course, radiological 

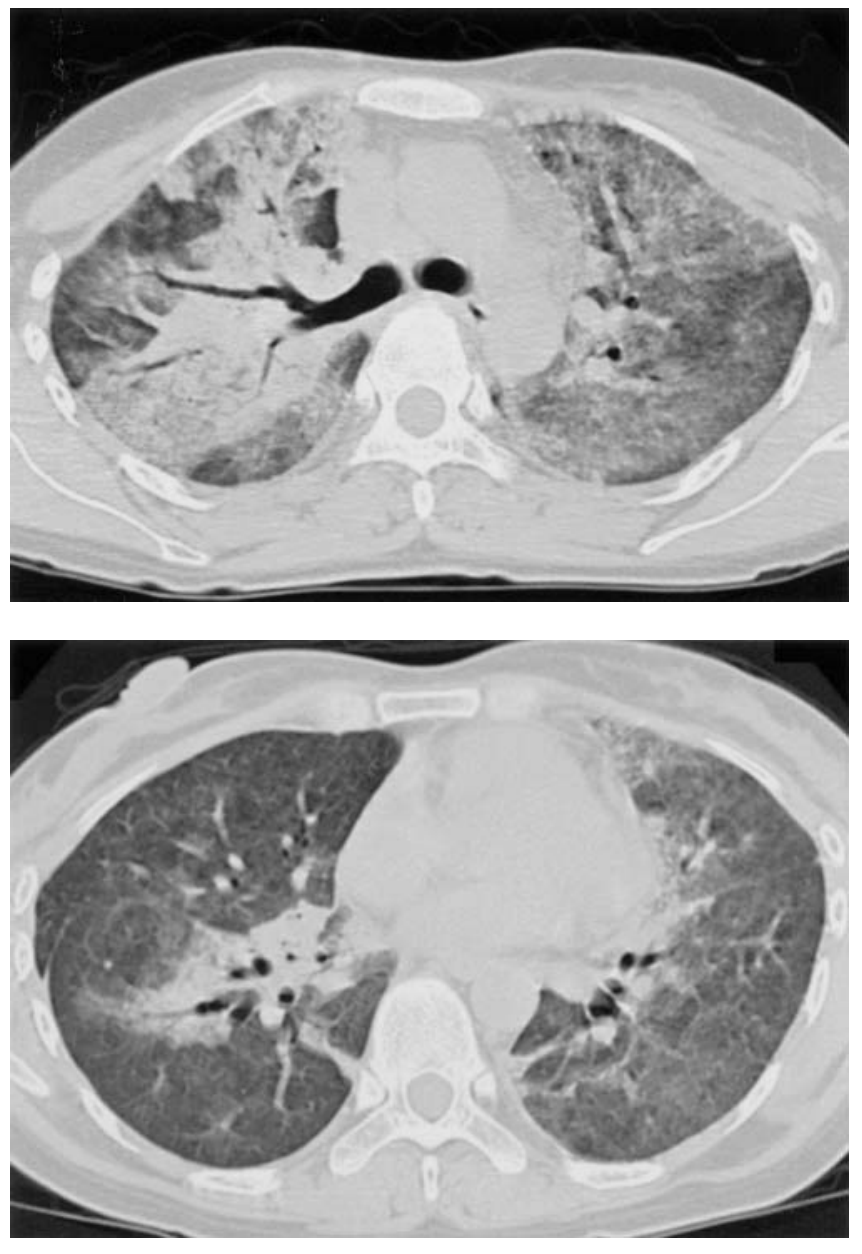

Figure 2. Computed tomographic (CT) scan of the thorax at time of admission shows bilateral areas of ground glass attenuation with areas of consolidation. Areas of opacity were observed diffusely, but seemed more prominent in the perihilar area.

findings, and if possible, pathological findings may give a clue to the type of pneumonitis involved.

Differential diagnosis would include atypical chest infections such as Pneumocystis carinii pneumonia and progressive rheumatoid interstitial lung disease, both of which are difficult to differentiate clinically from pneumonitis due to MTX. Plain chest radiography and laboratory data often do not provide enough data for diagnosis, and there may be instances of MTX pneumonitis and infection occurring simultaneously. A more accurate diagnosis may be provided by bronchoalveolar lavage (BAL) fluid; culture, staining, and cellular distribution in BAL fluid may help distinguish between pneumonitis, infection, and RA interstitial lung disease.

Noncardiogenic pulmonary edema (NCPE), a syndrome characterized by severe hypoxemia, bilateral alveolar infiltrates on chest radiograph, and no evidence of congestive heart failure, is reported as a rare complication of MTX therapy in high doses for cancer patients (6-10). Given that the initial differentiation between the types of MTX pneumonitis is difficult, the diagnosis of drug-induced NCPE would be an exercise of exclusion, and in most of the past reports on MTX-induced pulmonary edema, the diagnoses were made using the clinical course, radiologic findings, and in some, pathological findings (6-9). Most of these reports of pulmonary edema caused by MTX involved intrathecally administered MTX, and although the mechanism is still unknown, neurogenic pulmonary edema has been speculated as the cause for these past cases. Yet, one report is of a patient who had previously received intrathecal MTX, but succumbed to NCPE right after her oral MTX (8). It is known that MTX injected intrathecally can be absorbed systemically, possibly causing the pathologic process occurring in the lungs. It is also a known fact that anticancer therapy can induce NCPE regardless of the route of administration (11). Therefore, MTX at a low dose, although never reported previously, could be considered as a cause for NCPE.

Clinical presentation of NCPE features varying degrees of respiratory distress that may progress rapidly to respiratory failure. Severe dyspnea, cough, tachypnea, fatigue, and fever are some common symptoms. Typically anticancer therapyinduced NCPE presents with a low-grade fever, although a high fever would not rule out MTX as the causative agent since temperatures as high as $40^{\circ} \mathrm{C}$ have been reported with MTX pneumonitis (4). Chest radiograph shows the normalsized heart, while diffuse bilateral interstitial or alveolar infiltrates develop in the lung fields. General laboratory results are non-specific and represent the underlying disease process. Since these findings are non-diagnostic, information such as the time proximity to administration of the offending drug, radiograph findings, exclusion of heart dysfunction and any infectious, metabolic causes, and possible response to corticosteroids may further support a more accurate diagnosis (11).

Although there are no trials concerning the treatment for MTX pneumonitis, an initial discontinuation of the offending drug followed by corticosteroid administration seems to the established course of therapy. Discontinuation of MTX alone may show clinical improvement, but improvement of chest radiograph usually occurs over a course of several weeks (12). Use of corticosteroid therapy appears to accelerate the rate of recovery in most patients, and patients who are severely ill are usually candidates for corticosteroid therapy. These patients are usually given high-dose corticosteroid after-therapy, which are then tapered over the subsequent 2 to 4 weeks as determined by the patient's recovery (4). For most MTX pneumonitis immediate recovery is rare, even when corticosteroids are used, thus suggesting a certain degree of organic damage of the lungs. In contrast with druginduced pneumonitis that may cause tissue destruction, NCPE can be fully reversed upon early recognition, discontinuation of the causative drug and immediate start of intensive supportive treatment and intravenous corticosteroids. With proper treatment, improvement of the radiograph 


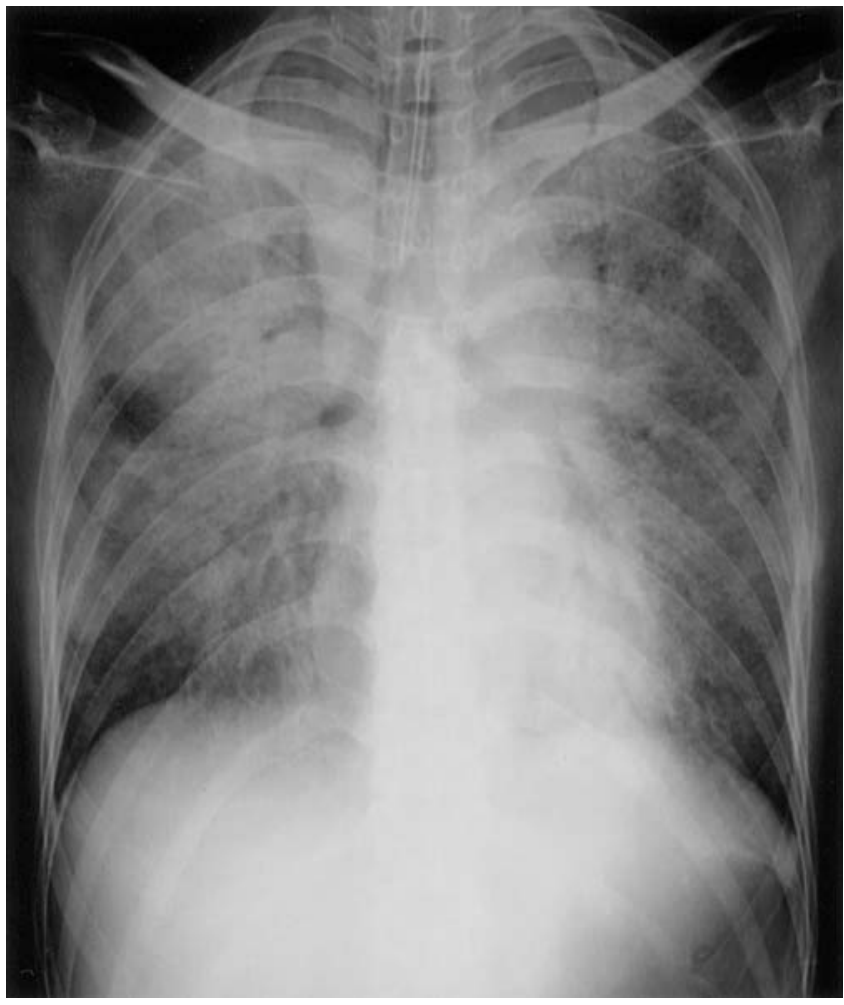

A

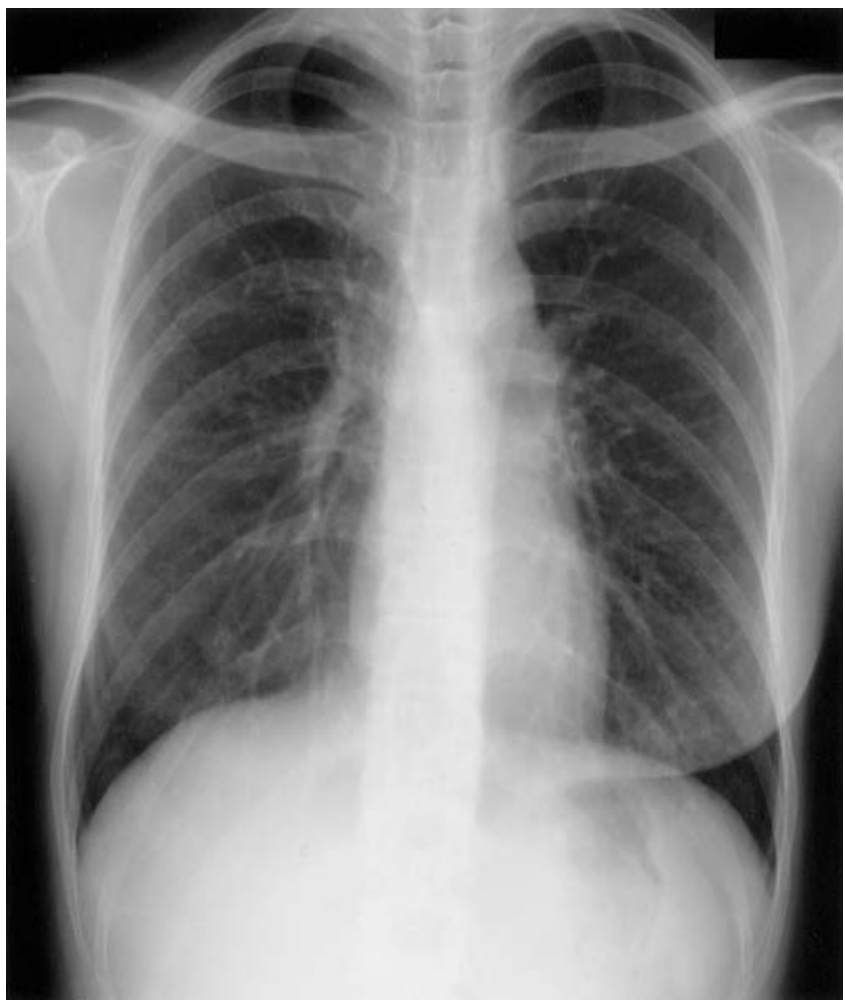

B

Figure 3. A) Chest radiograph on hospital day two, immediately after intubation. B) Chest radiograph on hospital day six after treatment. Complete recovery was observed at this point.

should occur within a short time span and prolonged corticosteroid therapy would be unnecessary.

In the present case, the clinical manifestations, radiological abnormalities, findings of bronchoalveolar lavage (marked lymphocytosis accompanied by marked elevation of CD4/8 ratio), and exclusion of infection led to the diagnosis of MTX-induced lung injury. The rapid and complete improvement of the disease with steroid pulse therapy alone in a short time period suggests that no organic lung damage had occurred. One would assume that the severity of our patient would usually require a more rigorous use of corticosteroid, but despite the patient's clinical symptoms, laboratory data, and clinical findings, respiratory failure rapidly recovered with only intravenous steroid and diuretics. Chest X-ray also exhibited this rapid recovery; this strongly suggests a reversible mechanism such as pulmonary edema. Although an echocardiogram was not performed, there were no clinical manifestations or laboratory data indicating any cardiac event that would have caused heart failure leading to pulmonary edema in this patient. Therefore, we conclude from our findings that the pathophysiology of this case was NCPE caused by low-dose MTX.

The exact mechanism of lung injury in the case of NCPE is still unknown. A direct toxicity of the drug to the alveolar type I pneumocytes or pulmonary vascular endothelial cells and the triggering of a cytokine-mediated inflammatory response is a popular hypothesis (11). These processes may result in increased vascular permeability accompanied by extravasation of fluids and proteins into the air spaces (13). We assume that in the present case, an activated immune mechanism, represented by the increased CD4+ lymphocyte counts in the BAL fluid, played some role in the increase of membrane permeability, causing pulmonary edema, and ultimately leading to respiratory failure.

Drug-induced pulmonary disease is a well-recognized complication of MTX treatment; it can be life threatening, and is sometimes fatal. It is important to take note, as in the present case, that weekly, low-dose MTX, although rare, may cause an acute form of lung toxicity, such as, NCPE. Prompt evaluation of the patient's clinical conditions and awareness of the possibility of NCPE is important for early detection, diagnosis, and treatment. In such cases, if not fatal, the condition can be fully reversed upon prompt recognition, discontinuation of the offensive drug and immediate start of intensive support treatment and intravenous corticosteroids. 


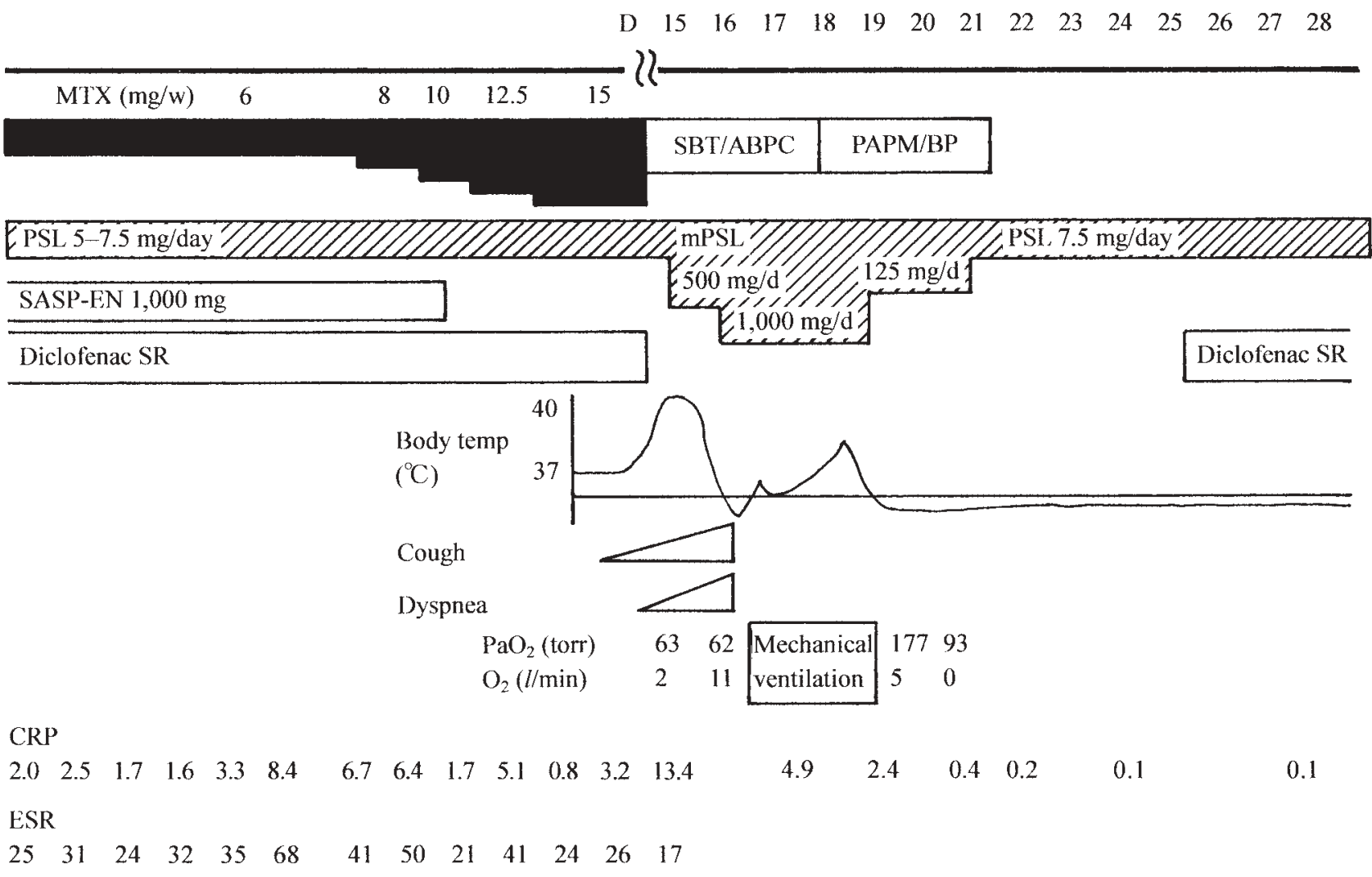

Figure 4. Clinical course. MTX: methotrexate, SBT/ABPC: sulbactam sodium/ampicillin, PAPM/BP: panipenem/betamipron, PSL: prednisolone, mPSL: methylprednisolone, CRP: C-reactive protein, ESR: erythrocyte sedimentation rate.

\section{References}

1) Tugwell P, Bennet K, Bell M, Gent M. Methotrexate in rheumatoid arthritis. Feedback on American College of Physicians Guidelines. Ann Int Med 110: 581-583, 1989.

2) Acute Leukemia Group B. Acute lymphocytic leukemia in children. Maintenance therapy with methotrexate administered intermittently. J Am Med Assoc 207: 923-928, 1969.

3) Sostman HD, Matthay RA, Putman CE, Smith GJ. Methotrexateinduced pneumonitis. Medicine 55: 371-388, 1976.

4) Cannon GW, Ward JR, Clegg DO, Samuelson CO Jr, Abott TM. Acute lung disease associated with low-dose pulse methotrexate therapy in patients with rheumatoid arthritis. Arthritis Rheum 26: 1269-1274, 1983.

5) Engelbrecht JA, Calhoon SL, Scherrer JJ. Methotrexate pneumonitis after low-dose therapy for rheumatoid arthritis. Arthritis Rheum 26: 1275-1278, 1983.

6) Bernstein ML, Sobel DB, Wimmer RS. Noncardiogenic pulmonary edema following injection of methotrexate into the cerebrospinal fluid. Cancer 50: 866-868, 1982.
7) Hamous JE, Guffy M, Aschenbrener CA. Fatal acute respiratory failure following intrathecal methotrexate administration. Cancer Treat Rep 67: 1025-1026, 1983.

8) Lascari AD, Strano AJ, Johnson WW, Collins JG. Methotrexateinduced sudden fatal pulmonary reaction. Cancer 40: 1393-1397, 1977.

9) Gutin PH, Green MR, Bleyer WA, Bauer VL, Wiernik PH, Walker MD. Methotrexate pneumonitis induced by intrathecal methotrexate therapy. A case report with pharmacokinetic data. Cancer 38: 15291534, 1976.

10) Reed CR, Glauser FL. Drug-induced noncardiogenic pulmonary edema. Chest 100: 1120-1124, 1991.

11) Briasoulis E, Pavlidis N. Noncardiogenic pulmonary edema: an unusual and serious complication of anticancer therapy. Oncologist 6: 153-161, 2001.

12) Searles G, McKendry RJ. Methotrexate pneumonitis in rheumatoid arthritis: potential risk factors. Four case reports and a review of literature. J Rheumatol 14: 1164-1171, 1987.

13) Flick MR, Matthay MA. Pulmonary edema and acute lung injury. In: Textbook of Respiratory Medicine, 3rd ed. Murray JF, Nadel JA, Eds. W.B. Saunders Company, Philadelphia, 2000: 1575-1598. 